\title{
Effects of coronary endarterectomy on postoperative early results in long segment coronary artery disease
}

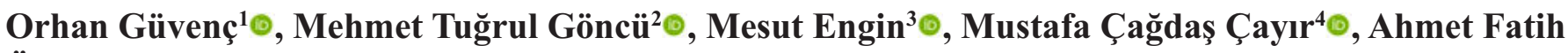 \\ Özyazıcıoğlu² $\odot$
}

\begin{abstract}
${ }^{1}$ Department of Cardiovascular Surgery, A $\breve{g r l}$ State Hospital, A $\breve{g r l}$, Turkey
${ }^{2}$ Department of Cardiovasculer Surgery, University of Health Sciences, Bursa Yüksek Ihtisas Training and Research Hospital, Bursa, Turkey

${ }^{3}$ Department of Cardiovasculer Surgery, University of Health Sciences, Şanliurfa Mehmet Akif Inan Training and Research Hospital, Şanlıurfa, Turkey

${ }^{4}$ Department of Cardiovasculer Surgery, Pamukkale University School of Medicine, Denizli, Turkey
\end{abstract}

\begin{abstract}
Objectives: Main goal of coronary bypass surgery is complete revascularization. In some coronary endarterectomy applied patient groups, complete revascularization is inevitable. In this study, it was aimed to reveal factors affecting early mortality and morbidity in patients undergoing coronary endarterectomy.

Methods: Retrospective records of preoperative, operative, and postoperative data of 98 patients undergoing coronary artery bypass grafting (CABG) with coronary endarterectomy between January 1, 2012, and October 30, 2016, were reviewed.

Results: A total of 113 endarterectomies were performed in different coronary arteries. Of the patients, 22 $(22.4 \%)$ were female and $76(77.6 \%)$ were male. The mean of ages was $60.4 \pm 9.9$ (range; 36-81 years). A positive inotropic requirement was required in $68(69.1 \%)$ patients and intra-aortic balloon pump was required for $23(23.4 \%)$ patients. Mortality was observed in 10 patients $(10.2 \%)$. Peroperative myocardial infarction was observed in $17(17.3 \%)$ patients. Mortality rate was significantly higher in patients whose left ventricular ejection fraction was 30 or less and who had a higher risk in EuroSCORE $(p<0.001)$. When compared with the other vessels, mortality ratewas found to be higher for left anterior descending coronary artery endarterectomy $(p=0.038)$. Mortality in female patients undergoing endarterectomy was higher than male patients $(p=$ $0.023)$.

Conclusions: Mortality and morbidity are higher in patients undergoing coronary endarterectomy when compared to conventional $\mathrm{CABG}$ operations. However, it is a method that can be applied by considering certain risk factors.
\end{abstract}

Keywords: Endarterectomy, bypass surgery, coronary artery

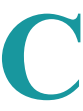
oronary artery diseases (CADs) are one of the most important causes of death in our age. Coronary Artery Bypass Grafting (CABG) surgery is still the most valuable treatment option in the treatment of CAD despite the improvements in cardiology. Com- plete revascularization is intended in CABG operations. The aim is to provide blood flow to the diseasefree region in the distal coronary artery. However, finding a disease-free zone is not possible in all operations. This is particularly important in patients with 
diffuse coronary disease. In this situation, it is necessary to remove the atheroma plaque from the coronary artery [1]. The frequency of this group of patients is reported between $0.8 \%$ and $25.1 \%$ [2].

The aim of this study was to investigate the early mortality and morbidity outcomes of patients undergoing elective coronary artery surgery.

\section{METHODS}

This study was performed in patients who underwent coronary endarterectomy between January 2012-October 2016, at Bursa Yüksek İhtisas Training and Research Hospital, Department of Cardiovasculer Surgery. The study protocol was approved by the local institutional Ethical Committee of University of Health Sciences. The patients were evaluated retrospectively and the effect of coronary endarterectomy on postoperative morbidity and mortality was evaluated with statistical methods.

Preoperative, operative and postoperative data of the patients were obtained by examining patient files and hospital information management system records. Patients who underwent CABG and no additional cardiac procedure were included in the study. Preoperative, intraoperative and postoperative data of patients were recorded and mortality and morbidity analyzes were performed.

\section{Surgical Endarterectomy Technique}

In patients who underwent closed (traction, pull out) endarterectomy technique; a small arteriotomy was performed in the coronary artery. The atheromatous lesion was dissected and taken out by traction. The arteriotomy and incision were continued until the atheromatous nucleus was reached and removed. If the incision was no longer needed, the bypass graft was anastomosed.

In patients undergoing open endarterectomy technique; with a long arteriotomy extending from the damaged vascular segment to the healthy vascular segment, it was ensured that the atheromatous nucleus was completely cleared from all the branches of the artery. Then, the arteriotomy was closed with internal thoracic artery (ITA) or saphenous vein graft. In some patients, arteriotomy was closed with saphenous vein patch and an ITA graft anastomosed on the patch.

\section{Statistical Analysis}

Statistical analysis data were analyzed with the Statistical Package for the Social Sciences (IBM SPSS Statistic Inc. version 16.0, Chicago, IL, USA). Continuous and ordinal variables were expressed as the mean \pm standard deviation and nominal variables were expressed as frequency and percentage. Kolmogorov-Smirnov test and Shapiro-Wilk tests of normality were used to identify the distribution of variables. Student's t-test was used to compare two groups for continuous variables with normal distribution. Mann-Whitney $U$ test was used to compare two groups for continuous variables without normal distribution. Chi-Square test was used to compare two groups for nominal variables. For all tests, a $p$-value of $<0.05$ was considered statistically significant.

\section{RESULTS}

Of the 98 patients included in the study, 22 $(22.4 \%)$ were female and $76(77.6 \%)$ were male. The mean age was $60.4 \pm 9.9$ (range; 36-81 years). More than half of the patients $(59.9 \%)$ were over 60 years old. There were $60(60.4 \%)$ patients with a family history of coronary artery disease, $59(60.2 \%)$ patients with anti-hypertensive treatment, and $44(45 \%)$ patients with diabetes mellitus (DM). Fifty-nine $(60.2 \%)$ of the patients had smoking habit. The body mass index of $32(33 \%)$ patients was over 30 . In the biochemical examination of the preoperative period, cholesterol and triglyceride levels of 71 patients were detected as above normal values (Table 1).

Fifty-seven (58.1\%) of the patients had previously undergone coronary intervention (coronary balloon and/or stent). In terms of left ventricular ejection fraction (LVEF), 21 (21.4\%) patients with LVEF $\leq$ $30 \%, 37(37.7 \%)$ patients with LVEF $30-50 \%$ range and $40(40.8 \%)$ patients with LVEF $\geq 50 \%$ were detected. The patients were classified for EuroSCORE as low risk (0-3 points), medium risk (4-6 points) and high risk ( 7 points and above) groups. There were 39 (39.7\%) patients with low risk, $48(48.9 \%)$ patients with medium risk and $11(11.2 \%)$ patients with highrisk. Fourteen $(14.2 \%)$ patients had a peripheral arterial disease (PAD), $33(33.6 \%)$ patients had a chronic obstructive pulmonary disease (COPD), 6 
Table 1. Demographic and clinic characteristics of the patients

\begin{tabular}{|c|c|}
\hline Characteristics & Data \\
\hline Age (years) & $60.3 \pm 9.9$ \\
\hline \multicolumn{2}{|l|}{ Gender } \\
\hline Male, n (\%) & $76(77.6)$ \\
\hline Female, n $(\%)$ & $22(22.4)$ \\
\hline CAD history in family, $\mathrm{n}(\%)$ & $60(61.2)$ \\
\hline Obesity, n (\%) & $32(32.6)$ \\
\hline DM, n (\%) & $44(44.8)$ \\
\hline Smoking, n (\%) & $59(60.2)$ \\
\hline Hyperlipidemia, n (\%) & $71(72.4)$ \\
\hline Hypertension, $\mathrm{n}(\%)$ & $59(60.2)$ \\
\hline COPD, n (\%) & $33(33.6)$ \\
\hline PAD, n (\%) & $14(14.2)$ \\
\hline CVA, n (\%) & $6(6.1)$ \\
\hline Previous PCI, n (\%) & $57(58.1)$ \\
\hline $\mathrm{EF} \leq 30, \mathrm{n}(\%)$ & $21(21.4)$ \\
\hline $\mathrm{EF}=30-50, \mathrm{n}(\%)$ & $37(37.7)$ \\
\hline $\mathrm{EF} \geq 50, \mathrm{n}(\%)$ & $40(40.8)$ \\
\hline EuroScore $=0-3, \mathrm{n}(\%)$ & $39(39.7)$ \\
\hline EuroScore $=4-6, \mathrm{n}(\%)$ & $48(48.9)$ \\
\hline EuroScore $=7$ and above, $\mathrm{n}(\%)$ & $11(11.2)$ \\
\hline CRF, n (\%) & $7(7.1)$ \\
\hline
\end{tabular}

$\mathrm{CAD}=$ Coronary artery disease, $\mathrm{DM}=$ Diabetes mellitus, $\mathrm{COPD}=$ Chronic obstructive pulmonary disease, $\mathrm{PAD}=$ Peripheral artery disease, CVA $=$ Cerobrovasculer accident, $\mathrm{PCI}=$ Percutaneur coronary intervention, $\mathrm{EF}=$ Ejection fraction, $\mathrm{CRF}=\mathrm{Chronic}$ renal failure

(6.1\%) patients had a cerebrovascular accident (CVA), and $7(7.1 \%)$ patients had chronic renal failure (CRF). Coronary endarterectomy was performed to provide complete revascularization during coronary bypass in our patient group. Minimum 1or maximum 2 endarterectomies were applied (mean: $1.1 \pm 0.3$ ). A total of 113 endarterectomy procedures were performed. Forty-one (36.2\%) were left anterior descending artery (LAD), 10 (8.8\%) diagonal (Dx), 1 $(0.8 \%)$ intermediary, $15(13.2 \%)$ circumflex $(\mathrm{Cx})$ and $46(40.7 \%)$ right coronary artery (RCA) (Table 2$)$.

In $15(15.3 \%)$ patients, two endarterectomies were performed at the same time. The distribution of these coronary endarterectomy vessels was found as 5
(4.4\%) LAD-Dx, 5 (4.4\%) LAD-Cx, 3 (2.6\%) LADRCA and $2(1.7 \%)$ Cx-RCA (Table 2). While the patients were transferring to intensive care unit, a positive inotropic requirement was provided in 68 (69.3\%) patients and intra-aortic balloon pump (IABP) support was provided in $23(23.4 \%)$ patients. The duration of mechanical ventilation was 6-120 hours (mean: $15.5 \pm 13.8$ ) in the postoperative period. The number of patients intubated over 48 hours was 5 . Total drainage volume of the patients in the first 48 hours in the intensive care unit was determined as minimum $300 \mathrm{ml}$, and maximum $1700 \mathrm{ml}$ (mean: 760 $\pm 280 \mathrm{ml}$ ). In postoperative intensive care follow-up, $52(53 \%)$ patients had various rhythm problems. The most common arrhythmia was atrial fibrillation and was seen in $37(37.7 \%)$ of the patients. This was followed by ventricular rhythm problems (ventricular extrasystole, ventricular tachycardia, ventricular fibrillation) with 15 (15.3\%) patients. These rhythm problems disrupt early postoperative hemodynamics. During the intensive care follow-up, 17 (17.3\%) patients had elevated blood cardiac enzyme levels (postoperative MI). Postoperatively, 17 (17.3\%) patients had elevation of liver function parameters and $19(19.3 \%)$ patients had impaired levels of renal function markers. Four patients (4\%) had cerebrovascular events and 2 of them had no previous complaints. The total length of stay of patients in the intensive care unit was 1-13 days (mean: $3.1 \pm 2$ days). The total hospital stay was 6-25 days (mean: $13.8 \pm$ 3.6) (Table 3).

Table 2. Endarterectomy data of the patients

\begin{tabular}{lc}
\hline & $\mathrm{n}(\%)$ \\
\hline RCA endarterectomy & $46(46.9)$ \\
LAD endarterectomy & $41(41.8)$ \\
Cx endarterectomy & $15(15.3)$ \\
Dx endarterectomy & $10(10.2)$ \\
İntermedier endarterectomy & $1(1)$ \\
LAD-Cx endarterectomy & $5(5.1)$ \\
LAD-Dx endarterectomy & $5(5.1)$ \\
LAD-RCA endarterectomy & $3(3)$ \\
Cx-RCA endarterectomy & $2(2)$ \\
\hline
\end{tabular}

RCA $=$ Right coronary artery, LAD $=$ Left anterior descending, $\mathrm{Cx}=$ Circumflex, Dx: Diagonal 
Table 3. Operative and postoperative data of the patients

\begin{tabular}{lc}
\hline Inotropic support, $\mathrm{n}(\%)$ & $68(69.1 \%)$ \\
IABP support, $\mathrm{n}(\%)$ & $23(23.4 \%)$ \\
Mean mechanical ventilation time (hours) & $15.5 \pm 13.8(6-120)$ \\
Mean total drainage volume in the first 48 hours (ml) & $760 \pm 280(300-1,750)$ \\
Arrhythmia, n (\%) & $52(53 \%)$ \\
Postoperative MI, n (\%) & $17(17.3 \%)$ \\
Posoperative impaired liver function, n (\%) & $17(17.3 \%)$ \\
Postoperative renal failure, n (\%) & $19(19.3 \%)$ \\
Postoperative CVE, n (\%) & $4(4 \%)$ \\
Mortality, n (\%) & $10(10.2 \%)$ \\
\hline
\end{tabular}

IABP = Intraaortic balloon pump, $\mathrm{CVE}=$ Cerebrovasculer event, $\mathrm{MI}=$ Myocardial infarction

Mortality was observed in $10(10.2 \%)$ patients. The highest mortality rate was in LAD endarterectomy group. Among these patients, 8 (8.1\%) with LAD and $1(1 \%)$ with LAD-RCA. The highest mortality rate was observed in female; $6(27.2 \%)$ deaths in female patients and $4(5.2 \%)$ deaths in male patients $(p=$ $0.023)$. Mortality in patients with moderate to low LVEF was significantly higher than in patients with normal LVEF. Mortality was observed in 10 patients with low LVEF $3(3 \%)$ and $7(7.1 \%)$ moderate patients. Mortality wasn't observed in normal LVEF group $(p<0.001)$. Mortality was observed in $6(6.1 \%)$ patients in the high-risk group, $4(4 \%)$ patients in the moderate risk group and in none of the patients in the lower risk group $(p<0.001)$. The mortality rate was $7.9 \%$ in patients who underwent LAD endarterectomy and $1.7 \%$ in the other patient group ( $p=0.038)$. Total ICU stay, total hospital stay and total perfusion times were observed as longer in patients with mortality (Table 4).

Table 4. Distribution of mortality according to various parameters

\begin{tabular}{|c|c|c|c|c|}
\hline \multicolumn{2}{|l|}{ 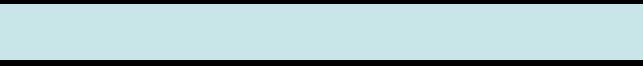 } & Survivors & Non-survivors & $p$ value \\
\hline \multicolumn{2}{|l|}{ Age (yeras) } & $59.4 \pm 10.3$ & $64.2 \pm 17.2$ & $0.381^{*}$ \\
\hline \multicolumn{2}{|c|}{ Total ICU stay (days) } & $3.1 \pm 1.9$ & $5.2 \pm 2.7$ & $0.032^{* *}$ \\
\hline \multicolumn{2}{|c|}{ Total hospital stay (days) } & $11 \pm 3.7$ & $17.7 \pm 6.6$ & $0.021^{* *}$ \\
\hline \multicolumn{2}{|c|}{$\mathrm{X}$-clamp time (min) } & $49.3 \pm 14.2$ & $65.7 \pm 28.1$ & $0.301^{* *}$ \\
\hline \multicolumn{2}{|c|}{ TPT (min) } & $89.4 \pm 15.8$ & $107.5 \pm 8.2$ & $0.007 * *$ \\
\hline \multirow{2}{*}{ Gender } & Female (n) & 16 & 6 & $0.023^{* * *}$ \\
\hline & Male (n) & 72 & 4 & \\
\hline \multirow[t]{2}{*}{ LVEF } & Low-Moderate (n) & 48 & 10 & $<0.001^{* * *}$ \\
\hline & Normal (n) & 40 & 0 & \\
\hline \multirow[t]{2}{*}{ EuroSCORE } & Low-Moderate (n) & 82 & 4 & $<0.001^{* * *}$ \\
\hline & $\operatorname{High}(\mathrm{n})$ & 5 & 6 & \\
\hline \multirow[t]{3}{*}{ Endarterectomy } & $\operatorname{LAD}(\mathrm{n})$ & 32 & 9 & $\mathbf{0 . 0 3 8}^{* * *}$ \\
\hline & RCA (n) & 44 & 2 & \\
\hline & Others (n) & 26 & 0 & \\
\hline
\end{tabular}

${ }^{*}$ Student-T test ${ }^{* *}$ Mann Whitney $\mathrm{U}$ test ${ }^{* * *}$ Chi-Square test. Data are shown are mean \pm standard deviation or number. ICU $=$ Intensive care unit, X-clamp time $=$ Cross-clamp time, TPT $=$ Total perfusion time, $\mathrm{LVEF}=\mathrm{Left}$ ventriculer ejection fraction 


\section{DISCUSSION}

Coronary endarterectomy was performed in 1957 by Bailey et al. [3], but no bypass was performed in those days. This method has always been discussed since the first implementation of this process to the present day. In the 1990s, this method was not recommended in routine practice because of high mortality and morbidity rates [4]. When we look at the literature, the rate of coronary endarterectomy application varies between 0.8 and $25 \%$ [5]. Considering that approximately $800 \mathrm{CABG}$ surgeries are performed annually at our center so this ratio is compatible with the literature.

In our study, the rate of male patients was $77.6 \%$. In many studies about coronary endarterectomy, the ratio of male patients was found to be high in accordance with our study [6]. The authors attributed this to the short diameters of the coronary arteries in female patients. In addition, in our study, we found that mortality rate in female patients who underwent coronary endarterectomy was higher with $27.2 \%$ compared to men with $5.2 \%$.

The diffuse disease of LAD has a special importance since LAD feeds a significant portion of the heart. The importance of revascularization of this vessel has been demonstrated in many studies [7]. The rate of LAD endarterectomy varies between $9 \%$ and $74.8 \%$ in the literature [8]. In our study, $36.2 \%$ of the patients underwent LAD endarterectomy. We observed that RCA was the most common endarterectomy vessel with $40.6 \%$. In the literature, this ratio varies between $21.1 \%$ and $83 \%[8,9]$. In some patients, multiple vascular endarterectomies may be required during the same operation. According to the literature, multiple coronary endarterectomies increase mortality [9]. In our study, we determined that two coronary endarterectomies were performed simultaneously in 15 patients. Of these, only two patients were treated with two vessels (RCA-Cx) except LAD.

Early extubation, intensive care, and less total hospital stay after open heart surgery are closely related to reduced mortality. In our study, the mean duration of intubation in the intensive care unit was 6120 hours. Takanashi et al. [8] reported that the duration of intubation was $12.6 \pm 18.7$ hours. The duration of intensive care stay and total hospitalization period ranged from 1 to 13,6 to 25 days, respectively. Schmitt et al. [10], found these durations as $5.6 \pm 8.4$ and $15.9 \pm 13.9$ days, respectively, in their coronary endarterectomy study.

Inotrope and IABP support may be required for avoiding from cardiopulmonary bypass after CABG operation. These need ratios may increase in patients undergoing coronary endarterectomy. In our study, the number ofa positive inotropic requirement was 68 $(69.3 \%)$ and IABP support was required in $23(23.4 \%)$ patients. Padhy et al. [11] statedthese rates as $64.2 \%$ and $16.6 \%$, respectively.

The amount of drainage of the patients after surgery affects the amount of blood and blood product transfer, which affects the mortality and morbidity. The total amount of drainage in our patients ranged from 300 to $1700 \mathrm{ml}$ (mean: $760 \pm 280 \mathrm{ml}$ ). Yener et al. [12], found the mean amount of mediastinal bleeding as $650 \pm 8.2 \mathrm{ml}$. Compared to this study, we can say that our drainage rates are acceptable.

Arrhythmias after open heart surgery may prolong hospital stay, serious arrhythmias can cause mortality and morbidity. Atrial fibrillation (AF) is the most common rhythm problem and ventricular arrhythmias can be seen less frequently. In our study, 52 (53\%) patients had various rhythm problems. The most common arrhythmia was AF in $37.7 \%$ of the patients. In the literature, this ratio varies between $25 \%$ and $30 \%[8,13]$. The treatment of this arrhythmia is very important for cardiac surgery. Because it increases the morbidity in the early period, If it is permanent, it paves the way for future complications.

One of the most important factors in early mortality and morbidity in patients undergoing coronary endarterectomy is peroperative MI. Although, the most common cause of per-operative MI is incomplete revascularization, residual material to be left in the vessel, intimal flap, vasospasm, air embolism, inadequate protection of myocardium under the cross-clamp and obstruction of the newly constructed bypass graft may cause [14]. The incidence of perioperative MI varies between 1.5\% and $19 \%$ [15]. This rate was found to be $17.3 \%$ in our study.

The mortality rate was $10.2 \%$ (10 patients) in our study. Nine of these ten patients had LAD endarterectomy. Especially, studies showing that mortality has increased in the patients who underwent 
LAD endarterectomy support our study [16]. The 10\% mortality rate we found in our study is very high when compared with the conventional surgical method. The studies in the literature are also in this direction. However, we can say that this increased mortality rate is affected by comorbid factors [17].

\section{CONCLUSION}

In conclusion, we suggest that coronary endarterectomy should be performed in the right patient group at the right time and with the right methods. We should consider this operation as a lifesaving technique in patients with the common CAD, which is thought to be inoperable. Otherwise, these patients will either face death or we will consider more severe treatment methods such as heart transplantation and heart support devices.

\section{Author Contribution}

All authors have directly participated in the planning, execution, analysis or reporting of this research paper. All authors have read and approved the final version of the manuscript.

\section{Conflict of interest}

The authors disclosed no conflict of interest during the preparation or publication of this manuscript.

\section{Financing}

The authors disclosed that they did not receive any grant during conduction or writing of this study.

\section{REFERENCES}

1. Nishigawa K, Fukui T, Takanashi S. Coronary endarterectomy for the diffusely diseased coronary artery. Gen Thorac Cardiovasc Surg 2014;62:461-7.

2. Soylu E, Harling L, Ashrafian H, Athanasiou T. Does coronary endarterectomy technique affect surgical outcome when combined with coronary artery bypass grafting? Interact
Cardiovasc Thorac Surg 2014;19:848-55.

3. Bailey CP, May A, Lemmon WM. Survival after coronary endarterectomy in man. JAMA 1957;164:641-6.

4. Shapira OM, Akopian G, Hussain A, Adelstein M, Lazar LH, Aldea GS, et al. Improved clinical outcomes in patients undergoing coronary artery bypass grafting with coronary endarterectomy. Ann Thorac Surg 1999;68:2273-8.

5. Ghatanatti R, Teli A. Coronary endarterectomy: recent trends. J Clin Diagn Res 2017;11:PE01-PE04.

6. Bagheri A, Masoumi A, Bagheri J. Early outcomes of coronary endarterectomy in patients undergoing coronary artery bypass surgery. Heart Surg Forum 2016;19:E059-63.

7. Gucu A, Goncu T, Yavuz S, Arican Özluk O, Eris C, Turk T, et al. Alternative option in patients with multisegmental left anterior descending coronary artery disease for providing complete myocardial revascularization. Int J Clin Exp Med 2014;7:142-7. 8. Takanashi S, Fukui T, Miyamoto Y. Coronary endarterectomy in the left anterior descending artery. J Cardiol 2008;52:261-8.

9. Stavrou A, Gkiousias V, Kyprianou K, Dimitrakaki IA, Challoumas D, Dimitrakakis G. Coronary endarterectomy: the current state of knowledge. Atherosclerosis 2016;249:88-98.

10. Schmitto JD, Kolat P, Ortmann P, Popov AF, Coskun KO, Friedrich $\mathrm{M}$, et al. Early results of coronary artery bypass grafting with coronary endarterectomy for severe coronary artery disease. J Cardiothorac Surg 2009;4:52.

11. Padhy K, Narasimham SBR, Murthy GSRC, Chaganti VR, Kumar PVVNM, Rao MB, et al. Coronary endarterectomy for diffuse extensive coronary artery disease. Ind $\mathrm{J}$ Thoracic Cardiovasc Surg 2005;21:251-5.

12. Yener Ümit A, Kervan Ü, Korkmaz K, Gedik Selçuk H, Budak Baran A, Genç Bahadır S, et al. The impact of coronary artery endarterectomy on mortality and morbidity during coronary artery bypass grafting. Turk Gogus Kalp Dama 2014;22:734-41.

13. Byrne JG, Karavas AN, Gudbjartson T, Leacche M, Rawn JD, Couper GS, et al. Left anterior descending coronary endarterectomy: early and late results in 196 consecutive patients. Ann Thorac Surg 2004;78:867-73.

14. Sirivella S, Gielchinsky I, Parsonnet V. Results of coronary artery endarterectomy and coronary artery bypass grafting for diffuse coronary artery disease. Ann Thorac Surg 2005;80:173844.

15. Fuster V. Epidemic of cardiovascular disease and stroke: the three main challenges. Circulation 1999;99:1132-7.

16. Jones EL, Weintraub WS. The importance of completeness of revascularization during long-term follow-up after coronary artery operations. J Thorac Cardiovasc Surg 1996;112:227-37.

17. Tiruvoipati R, Loubani M, Lencioni M, Ghosh S, Jones PW, Patel RL. Coronary endarterectomy:impact on morbidity and mortality when combined with coronary artery bypass surgery. Ann Thorac Surg 2005;79:1999-2003. 